(2) Open Access Full Text Article

\title{
Impaired cognition in geriatric patients with relation to earlier life mood disorder and traumatic brain injury: a hypothesis
}

This article was published in the following Dove Press journal:

Neuropsychiatric Disease and Treatment

\author{
Edward $\mathrm{H}$ Tobe \\ Department of Psychiatry, Cooper \\ Medical School of Rowan University, \\ Camden, NJ, USA
}

\begin{abstract}
The challenges of the geriatric years require cognitive integrity through organic resilience of the brain. Impaired cognition in geriatric patients (age $>65$ years) is commonly ascribed to age but is multifactorial. Among those multiple factors this author hypothesizes that mood disorders, with major depressive disorder (MDD) as one focus of this paper and traumatic brain injury (TBI) are part of a common spectrum of pathology that, when undiagnosed and untreated at age $<65$ years, reduces the resilience of the brain to negotiate common challenges during geriatric years. Mood disorders and TBI may be acute, transient, and benign; however, chronic mood disorders may be an organic brain disease, as shown by objective studies. The consequence of the ineffective treatment of MDD and TBI at an earlier age may cause geriatric patients to have impaired capacity to manage stressors. The solution may include more astute observation of the presentation to enable earlier diagnosis and treatment. Mitigating the consequences of mood disorders and TBI may enable greater resilience to face the challenges of aging.
\end{abstract}

Keywords: brain resilience, cognitive impairment, traumatic brain injury, mood disorders

\section{Introduction}

Mood disorders, major depressive disorder (MDD) and traumatic brain injury (TBI) usually are not referred to as diseases, but as disorders or syndromes. Some syndromes may cause transient disturbances without long-term sequelae. The etiologic heterogeneity of MDD and TBI might be a factor against the recognition of the importance of MDD and TBI as diseases with long-term impairment.

A disease may be defined as "a cause of discomfort or distress, a trouble, an annoyance, a grievance, a condition of the body or of some part or organ of the body in which its functions are disturbed or deranged; a morbid physical condition, a departure from the state of health especially when caused by structural change"1; "an impairment of the normal state of the living animal or plant body or of any of its components that interrupts or modifies performance of the vital functions", "literally the lack of ease; a pathological condition of the body that presents a group of symptoms peculiar to it and that sets the condition apart as an abnormal entity different from other normal or pathological body states; Syn. dyscrasia;"3 and "Dyscrasia: [Gr. Dyscrasia, bad temperament] an old term meaning abnormal mixtures of the four humors." ${ }^{4}$ These definitions of disease include chronic sequelae of mood disorders and TBI. Furthermore, mood disorders and TBI have
Correspondence: Edward $\mathrm{H}$ Tobe Department of Psychiatry, Cooper Medical School of Rowan University, I00I

Lincoln Drive West, Suite B, Marlton,

Camden, NJ 08053-1534, USA

Tel + I 8569834940

$\mathrm{Fax}+18569833408$

Email drtobel@gmail.com 
objective findings that are demonstrable with functional imaging and postmortem histology. Syndrome or disorder is based upon a group of signs and symptoms that occur jointly but may not have a pathological result.

MDD and TBI may have diverse presentations, based on the varied brain regions of injury and dysfunction. Some patients have remarkable resilience and are not as vulnerable to the long-term negative consequences of MDD or TBI. The extent of vulnerability may be a function of genetic predisposition, nuclear DNA, and mitochondrial DNA (mtDNA) that may provide adequate physiologic reserve in a delicately balanced intracellular enzymatic system to manage diseases.

In this article, MDD and TBI are discussed together because of the common consequences of both diseases. These consequences include potential chronic brain diseases, long-term loss of brain tissue resilience, and limited ability to manage the common stressors of geriatric life. It is hypothesized that MDD and TBI are part of a common spectrum of pathology that, when undiagnosed and untreated at age $<65$ years, reduces the resilience of the brain to negotiate common challenges during geriatric years. The purpose of this article is to review MDD and TBI in consideration of this hypothesis, and to suggest treatment options for these diseases.

\section{Mood disorder}

Byers et al, ${ }^{5}$ review numerous studies that suggest a correlation between early and late life depression and dementia. Byers offers possible causes of the correlation of depression to a greater incidence of dementia: vascular disease; alterations in glucocorticoid steroids and hippocampal atrophy; increased deposition of $\beta$-amyloid plaques; inflammatory changes; and deficits of nerve growth factors or neurotrophins. However, there is evidence that depression may be organic before the development of dementia.

Clinical observation has suggested that despite extensive psychological treatment, some individuals cannot overcome the organic character of their mental illness. This observation was noted by Anna Freud in the 18th Freud Anniversary Lecture of the New York Psychoanalytic Institute, when she stated, "as far back as 1937 Freud and Strachey had concluded that in some unanalyzable patients obstacles "are of the physiological and biological nature" and "in the main unsusceptible to psychological influences.",6,8 Anna Freud had referred some of her patients for psychotropic treatment during their analysis. This information was published by Morris Lipton to illustrate Anna Freud's concern for the patient, rather than the method. ${ }^{6,7}$
Extensive scientific data including histology substantiate the hypothesis that mood disorders may be associated with serious biological residuals. ${ }^{9}$ The biological residuals may be proportional to the duration of the MDD, even though the patient may have had mood recovery before death. ${ }^{9}$ The recognition of this abnormal histology was known and debated in the late 1800s. Mairet observed postmortem changes in the temporal lobe of melancholic patients and hypothesized that "this area might be related to primary feelings of sadness and that the nihilistic delusions were in fact secondary developments made possible by the spread of the lesion to the cortex." ${ }^{10}$ Histologic analyses from more recent studies confirmed neuronal and glial cell changes of the left caudal orbitofrontal region and dorsolateral prefrontal cortical region in patients who had major depression. ${ }^{11}$ A histological study of 60 postmortem brain tissue specimens from area $24 \mathrm{~b}$ of the supracallosal anterior cingulate cortex, separated into 4 groups of 15 subjects each with diagnoses that included MDD, bipolar disorder, schizophrenia, and normal control subjects, distinguished the MDD subjects by lower glial cell density (22\%; $P=0.004)$ and neuronal size $(23 \% ; P=0.01)$ in layer 6 than control subjects. ${ }^{12}$ When compared to controls there was some supportive evidence that the schizophrenia group showed reduced glial density in layer 6 but without changes of neuronal size.

\section{Traumatic brain injury}

The United States Centers for Disease Control and Prevention defines TBI as a disruption in normal brain function that may be caused by a seemingly minor or major blow or penetrating injury to the head. ${ }^{13}$

Those who survive a TBI can face effects that last a few days, or the rest of their lives. Effects of TBI can include impaired thinking or memory, movement, sensation (e.g., vision or hearing), or emotional functioning (e.g., personality changes, depression). These issues not only affect individuals but can have lasting effects on families and communities. $^{13}$

The military services have recognized the effect of blast injuries causing TBI, chronic encephalopathy, and dementia. ${ }^{14,15}$ The prevalence of TBI is difficult to assess because the causes of TBI are common and diverse including sports injuries, motor vehicle accidents, and falls in the home. Often the patient may perceive the injury as benign, "shake it off" and continue with normal activities not identifying the insult to the brain. Mental status examinations need to not merely ask if there has been a closed 
head injury but to actively seek a history of signs and symptoms such as anosmia, impaired cognition, and fatigue.

TBI may have cumulative effects on brain tissue and function. Adults aged $\geq 75$ years have the highest rates of hospitalization and deaths related to TBI of all age groups. ${ }^{16}$ The cumulative effects of brain trauma may appear minor initially, but may become important in the long term, even without additional injury. Frontal lobe injury may cause injured people to be oblivious to their own illness.

Identifying TBI may be difficult, in part because of the incorrect assumption that minor injuries cannot cause TBI. The residua of TBI increases the risk of early expression of neuropsychiatric disorders especially in the presence of a genetic load such as: synucleinopathy and/or tauopathy.

\section{Challenges to resilience}

As people age, there are many potential, often unforeseen, challenges of tissue resilience, including acute and chronic illness, loss of loved ones, socioeconomic compromise, loss of dignity, perceived or actual treatment as a societal burden, and placement in facilities for elderly care. In addition, people may face many hardships globally including war, starvation, and natural disasters. Vulnerability to the effects of MDD or TBI may be greater in people with a genetic profile of diseases such as neurodegenerative diseases. Resilience requires a level of physiologic reserve that may be difficult to quantify.

In general, metabolism creates energy by converting carbohydrates and fats into smaller units and synthesizes macromolecules such as proteins. Byproducts of metabolism include the development of reactive oxygen species that may damage macromolecules. The balance between damage and repair may be more important for aging than the absolute rate of damage and repair. ${ }^{17}$

Patients who have pathology caused by MDD or TBI may have greater challenges to resilience. When such challenges occur, predictable emotional changes may begin, with regression to primitive defenses. Elderly patients who have a history of MDD may complain of difficulty with auditory memory.

Physiologic resilience may be affected especially by mtDNA, that is composed of maternally inherited circular haploid groups that are named from $\mathrm{A}$ to $\mathrm{Z}$ in the sequence of their discovery. If mtDNA becomes compromised, cascades of dysfunctional metabolism may impair cellular connectivity and communication. In a study of 150 severely ill patients who had sepsis and were admitted to an intensive care unit, survival at 180 days was 2.12-fold greater in patients with than without haploid group $\mathrm{H}$. Therefore, genetic haploids may improve physiologic reserve that is protective against physiologic stress. ${ }^{18}$

\section{Underestimation of mood disorders and TBI}

Major depression is a heterogeneous class of disorders with diverse presentations determined by the areas of the brain affected. Treatment of mood disorders is often unsuccessful, and patients may have no improvement in their ability to function in average daily vocational and avocational aspects of life. Treatment is often managed with medical checks every few weeks or months, the condition may be trivialized, even though it prevents the body or mind from working properly.

In contrast, when a patient presents to the emergency department with a panic attack, an extensive cardiac evaluation may be pursued without psychiatric consultation. When the cardiac evaluation is negative, there typically is no parallel psychiatric evaluation performed. Instead, a false discharge diagnosis of atypical chest pain is provided. In these cases, the patient has lost the opportunity to be educated about the benefit of further evaluation and possible treatment to increase long-term resilience.

\section{Treatment options}

Physicians and other health care providers might reconsider the negative long-term outcome of common diseases such as MDD and TBI. When patients with a preexisting history of MDD or TBI enter the geriatric years, clinical findings may be observed including impaired resilience, cognitive deterioration, and diminished adaptation. Effective treatment of MDD and TBI may result from a careful diagnosis and treatment approach that is directed toward remission. A well-considered treatment strategy is an art that includes pharmacologic and psychotherapeutic care. First generation antidepressants such as tricyclic antidepressants (TCAs) and monoamine oxidase inhibitors (MAOIs) are effective, even though they have been underused by practitioners. Due to a lack of training about TCAs and MAOIs, there has been an over reliance on well-marketed drugs. The repetitive use of a single or two classes of drugs, such as the selective serotonin reuptake inhibitors and the serotonin norepinephrine reuptake inhibitors despite minimal benefit, may diminish the patient's quality of life and increase the risk of tachyphylaxis. 
TBI needs prudent following to determine if there are changes of mood, cognition, personality. The assumption of a benign injury can be quite misleading. ${ }^{18}$ Early intervention with cognitive adaptations diminishes frustration. Medications may include: direct stimulants, glutamate partial agonists, acetylcholine esterase inhibitors, and selegiline, in low dose a selective MAO type B inhibitor. Dopamine blocking agents have a risk if there is basal ganglion metabolic change, thus the advantage of FDG PET of the brain. ${ }^{19}$

\section{Summary}

The meaning of geriatric years may vary by longevity of individuals within a culture. What is certain are the challenges of becoming old. There may be struggles with economic limitations, access for health care, loss of friends logistically or through death, loss of capacity to sustain gainful employment. Often this is a phase of increased medical comorbidities. These challenges require cognitive integrity through organic resilience of the brain. Untreated TBI and chronic mood disorder are diseases that deplete the brain as an organ from providing optimal resilience at a time of vulnerability.

\section{Disclosure}

The author reports no conflicts of interest in this work.

\section{References}

1. Weiner ESC, Simpson JA, editors. The Compact Oxford English Dictionary: Second Edition, Complete Text. New York: Oxford University Press; 1993.

2. Onions CT, Friedrichsen GW, Burchfield RW, editors. Oxford Dictionary of English Etymology. Oxford: Oxford University Press; 1966.

3. Gove PB, editors. Webster's Third New International Dictionary, Unabridged. Chicago: Encyclopaedia Britannica; 1981.

4. Thomas CL, editors. Taber's Cyclopedic Medical Dictionary 15th Edition. Philadelphia: FA Davis; 1985.

5. Byers AL, Yaffe K. Depression and risk of developing dementia. Nat Rev Neurol. 2011:7(6):323-331. Published 2011 May 3. doi:10.1038/ nrneurol.2011.60.
6. Freud A. Difficulties in the Path of Psychoanalysis. New York: International Universities Press; 1969.

7. Lipton MA. A letter from Anna Freud. Am J Psychiatry. 1983;140 (12):1583-1584. doi:10.1176/ajp.140.12.1583

8. Freud S. Analysis terminable and interminable (1937). In: Freud S, editor. Complete Psychological Works. Standard. Vol. 23. London: Hogarth Press; 1964:212.

9. Tobe EH. Mitochondrial dysfunction, oxidative stress, and major depressive disorder. Neuropsychiatr Dis Treat. 2013;9:567-573. doi:10.2147/NDT.S44282

10. Berrios GE. "Depressive pseudodementia" or "melancholic dementia": a 19th century view. J Neurol Neurosurg Psychiatry. 1985;48 (5):393-400. doi:10.1136/jnnp.48.5.393

11. Rajkowska G, Miguel-Hidalgo JJ, Wei J, et al. Morphometric evidence for neuronal and glial prefrontal cell pathology in major depression. Biol Psychiatry. 1999;45(9):1085-1098.

12. Cotter D, Mackay D, Landau S, Kerwin R, Everall I. Reduced glial cell density and neuronal size in the anterior cingulate cortex in major depressive disorder. Arch Gen Psychiatry. 2001;58 (6):545-553.

13. Centers for Disease Control and Prevention (CDC). Traumatic brain injury \& concussion. CDC Web site; 2019 March 4. https://www.cdc.gov/traumaticbraininjury/index.html. Accessed March 7, 2018.

14. McKee AC, Robinson ME. Military-related traumatic brain injury and neurodegeneration. Alzheimers Dement. 2014;10(suppl 3):S242S253. doi:10.1016/j.jalz.2014.04.003

15. Hasoon J. Blast-associated traumatic brain injury in the military as a potential trigger for dementia and chronic traumatic encephalopathy. US Army Med Dep J. 2017;1-17:102-105.

16. Centers for Disease Control and Prevention (CDC). Report to congress. Traumatic Brain Injury in the United States: Epidemiology and Rehabilitation. Atlanta: National Center for Injury Prevention and Control, Division of Unintentional Injury Prevention; 2015. CDC Web site. Available from: https://www.cdc.gov/traumaticbraininjury/ pdf/tbi_report_to_congress_epi_and_rehab-a.pdf. Accessed March 7, 2019.

17. van Beek JH, Kirkwood TB, Bassingthwaighte JB. Understanding the physiology of the ageing individual: computational modelling of changes in metabolism and endurance. Interface Focus. 2016;6 (2):20150079. doi:10.1098/rsfs.2015.0079

18. Baudouin SV, Saunders D, Tiangyou W, et al. Mitochondrial DNA and survival after sepsis: a prospective study. Lancet. 2005;366 (9503):2118-2121. doi:10.1016/S0140-6736(05)67890-7

19. Tobe EH. Functional imaging: a necessary prerequisite to neuropsychological assessment. Int $J$ Diagn Imaging. 2014;1(2):74. doi:10.5430/ijdi.v1n2p74
Neuropsychiatric Disease and Treatment

\section{Publish your work in this journal}

Neuropsychiatric Disease and Treatment is an international, peerreviewed journal of clinical therapeutics and pharmacology focusing on concise rapid reporting of clinical or pre-clinical studies on a range of neuropsychiatric and neurological disorders. This journal is indexed on PubMed Central, the 'PsycINFO' database and CAS, and is the official journal of The International Neuropsychiatric Association (INA). The manuscript management system is completely online and includes a very quick and fair peer-review system, which is all easy to use. Visit http://www.dovepress.com/testimonials.php to read real quotes from published authors. 